SMAD, Rev. Eletrônica Saúde Mental Álcool Drog.

2019 jul.-set.;15(3):1-9

DOI: 10.11606/issn.1806-6976.smad.2019.148973

www.revistas.usp.br/smad/

Artigo Original

\title{
Uso de substâncias psicoativas entre estudantes universitários
}

\author{
Dominick Danielle Mendonça Santos ${ }^{1,2}$ \\ (D) https://orcid.org/0000-0002-5630-2986 \\ Melissa Monteiro Guimarães ${ }^{1}$ \\ (D) https://orcid.org/0000-0001-6773-4920 \\ Emerson Cotta Bodevan ${ }^{3}$ \\ (D) https://orcid.org/0000-0001-7471-9956 \\ Ricardo Lopes Rocha ${ }^{1}$ \\ (ID) https://orcid.org/0000-0002-6252-5246 \\ Marcos Luciano Pimenta Pinheiro ${ }^{1}$ \\ (DD https://orcid.org/0000-0001-9939-1045
}

1 Universidade Federal dos Vales do Jequitinhonha e Mucuri, Faculdade de Ciências Biológicas e da Saúde, Diamantina, MG, Brasil.

2 Superintendência Regional de Saúde de Diamantina, MG, Brasil.

${ }^{3}$ Universidade Federal dos Vales do Jequitinhonha e Mucuri, Faculdade de Ciências Exatas, Diamantina, MG, Brasil.
O objetivo do estudo foi identificar o perfil do uso de substâncias psicoativas entre estudantes dos cursos de Ciências Biológicas, Educação Física, Enfermagem, Fisioterapia, Farmácia, Odontologia e Nutrição. Trata-se de estudo transversal, quantitativo, exploratório e descritivo, com 567 estudantes. Os dados foram coletados por meio de um questionário e analisados através da estatística descritiva. Nos resultados, verificou-se que $11,6 \%$ dos estudantes nunca experimentaram substâncias psicoativas na vida. As drogas mais prevalentes entre os universitários foram o álcool $(n=501)$, tabaco $(n=161)$, maconha $(n=115)$ e cocaína $(n=26)$. Conclui-se que o consumo de substâncias psicoativas entre os estudantes é problema que deve ser enfrentado através da implantação de ações preventivas e de redução de danos.

Descritores: Saúde Mental; Atenção Primária a Saúde; Estratégia de Saúde da Família; Saúde da Família.

\section{Como citar este artigo}

Santos DDM, Guimarães MM, Bodevan EC, Rocha RL, Pinheiro MLP. Use of psychoactive substances among university students. SMAD, Rev Eletrônica Saúde Mental Álcool Drog. 2019;15(3):1-9. doi: https://dx.doi.org/10.11606/issn.1806-6976.smad.2019.148973 


\section{Use of psychoactive substances among university students}

The objective of this study was to identify the profile of use of psychoactive substances among students of Biological Sciences, Physical Education, Nursing, Physiotherapy, Pharmacy, Dentistry and Nutrition courses. It was a cross - sectional, quantitative, exploratory and descriptive study with 567 students. Data were collected through a questionnaire and analyzed through descriptive statistics. In the results, it was found that $11.6 \%$ of students had never tried psychoactive substances in their lives. The most prevalent drugs among university students were alcohol $(n=501)$, tobacco $(n=161)$, marijuana $(n=115)$, and cocaine $(n=26)$. The result led to the conclusion that consumption of psychoactive substances among students is a problem that must be faced through the implementation of preventive and harm reduction actions.

Descriptors: Mental Health; Primary Health Care; Family Health Strategy; Family Health.

\section{Uso de sustancias psicoactivas entre estudiantes universitários}

El objetivo del estudio fue identificar el perfil del uso de sustancias psicoactivas entre estudiantes de Ciencias Biológicas, Educación Física, Enfermería, Fisioterapia, Farmacia, Odontología y Nutrición. Se trata de un estudio transversal, cuantitativo, exploratorio y descriptivo, con 567 estudiantes. Los datos fueron recolectados por medio de un cuestionario y analizados a través de la estadística descriptiva. En los resultados, se constató que el 11,6\% de los estudiantes nunca experimentó el consumo de sustancias psicoactivas. Las drogas prevalentemente consumidas fueron: alcohol $(n=501)$, tabaco $(n=161)$, marihuana $(n=115)$ y cocaína $(n=26)$. Se concluye que el consumo de sustancias psicoactivas entre los estudiantes es un problema y que debe ser enfrentado a través de acciones preventivas y de reducción de daños.

Descriptores: Salud Mental; Atención Primaria de Salud; Estrategia de Salud Familiar; Salud de la Familia. 


\section{Introdução}

O abuso no consumo de substâncias psicoativas tornou-se preocupante para a sociedade e para a comunidade científica nas últimas décadas do século $\mathrm{XX}$. Seu consumo, cada vez mais precoce, tem apresentado altas taxas de incidência e prevalência e tem sido associado a graves riscos à saúde em decorrência de seu uso(1).

Muitos jovens no período da adolescência, com a vontade de tornar-se independente da família, procuram experimentar as drogas. Essa etapa do desenvolvimento humano é marcada por profundas mudanças tanto físicas quanto psíquicas, as quais tornam o adolescente mais vulnerável do ponto de vista psicológico e social, favorecendo uma maior exposição a fatores de risco, os quais podem facilitar, por exemplo, a inserção no uso de substâncias psicoativas(2).

O ingresso na universidade, ainda que traga sentimentos positivos e de alcance de uma meta programada por estudantes do ensino médio, por vezes pode se tornar um período crítico, de maior vulnerabilidade, que pode gerar o início e a manutenção do uso de álcool e/ou outras drogas(3).

Por conseguinte, o consumo de drogas começa a causar impactos já na vida acadêmica, resultando em falta de atenção durante as aulas, ausências, atrasos e maior sonolência(4). Esse problema está correlacionado ao crescimento da criminalidade, a acidentes automotivos, a comportamentos antissociais e à evasão escolar ${ }^{(5)}$.

Dada a alta prevalência do uso de drogas entre estudantes universitários e seu impacto na vida das pessoas e no desenvolvimento dos países, esse fenômeno tem despertado o interesse de pesquisadores no que diz respeito à sua identificação, compreensão e busca de soluções que possam intervir diretamente no bem-estar social ${ }^{(1)}$.

Em uma pesquisa de revisão acerca do consumo dessas substâncias entre estudantes universitários, no período de 1997 e $2007^{(6)}$, encontram-se os estudos de prevalência realizados em diversas universidades brasileiras com universitários de Medicina(7), Enfermagem ${ }^{(8)}$, Farmácia(9). Em todos esses estudos, o álcool foi a substância com maior prevalência de uso seguida pelo tabaco. Da mesma forma, dados do I Levantamento Nacional sobre o drogas de abuso entre universitários das 27 capitais brasileiras ${ }^{(3)}$, mostraram que as drogas utilizadas com maior frequência foram álcool $(86,2 \%)$ e tabaco $(46,7 \%)$.

Conhecer o padrão de consumo de substâncias psicoativas de determinada população, além de eliminar mitos existentes sobre o assunto, é essencial à implantação de programas de prevenção ao consumo de drogas e possibilita um direcionamento para o desenvolvimento de políticas públicas e de cuidado à saúde, incluindo sua prevenção e tratamento tanto no contexto geral como no contexto particular da universidade ${ }^{(5)}$. A escassez de dados que contemplem essa questão no Brasil tem sido motivo de novas pesquisas cujo foco é a busca e desenvolvimento de uma sociedade mais consciente e livre do uso abusivo dessas substâncias ${ }^{(5)}$.

O objetivo deste estudo foi traçar o perfil dos usuários de substâncias psicoativas entre os universitários da área da saúde da UFVJM e fornecer subsídios aos futuros programas de prevenção voltados para os mesmos.

\section{Método}

Trata-se de um estudo de desenho transversal, com abordagem quantitativa, exploratória e caráter descritivo realizado na Universidade Federal dos Vales do Jequitinhonha e Mucuri (UFVJM), campus de Diamantina, no período de março de 2013 a março 2014, com estudantes matriculados nos cursos de graduação da Faculdade de Ciências Biológicas e da Saúde (FCBS), a saber: Ciências Biológicas, Educação Física, Enfermagem, Fisioterapia, Farmácia, Odontologia e Nutrição.

A pesquisa foi previamente aprovada pelo Comitê de Ética em Pesquisa do CEP-UFVJM por meio do Processo no 075/2012.

O estudo contou com uma amostragem estratificada proporcional por cursos, considerando-se o grau de confiabilidade de $95 \%$, a precisão de $5 \%$ calculada com o auxílio dos dados de prevalência de um estudo

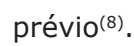

Os que aceitaram participar do estudo assinaram o Termo de Consentimento Livre e Esclarecido (TCLE) e receberam informações acerca dos objetivos, metodologia, bem como dos riscos e benefícios da pesquisa.

Para a coleta de dados, empregou-se um questionário estruturado e fundamentado em um estudo realizado entre os universitários das 27 capitais brasileiras ${ }^{(10)}$, contendo 24 questões de autopreenchimento referentes a dados sociodemográficos e sobre o uso das seguintes substâncias psicoativas: álcool, tabaco, maconha, cocaína, crack, tranquilizantes, inalantes e ecstasy, e o mesmo foi aplicado em horários de acordo com a disponibilidade relatada pelos respectivos coordenadores dos cursos.

Realizou-se um treinamento da equipe com o objetivo de avaliar o tempo gasto para responder ao questionário, capacitação dos pesquisadores e adequação do instrumento, sendo necessário o ajuste da questão 15 para melhorar sua compreensão. O tempo médio para o preenchimento do instrumento de pesquisa foi de 20 minutos. 
Os dados foram tabulados e a análise dos dados foi realizada por meio do programa $\mathrm{R}$ v. 2.14.1.

\section{Resultados}

De acordo com os dados da Tabela 1, participaram do estudo 567 universitários distribuídos nos cursos de Ciências Biológicas ( $n=76 ; 13.4 \%)$, Educação Física $(n=73 ; 12.9 \%)$, Enfermagem $(n=49 ; 8.6 \%)$, Farmácia $(n=102 ; 18.0 \%)$, Fisioterapia $(n=98 ; 17.3 \%)$, Nutrição $(n=67 ; 11.8 \%)$ e Odontologia $(n=102 ; 18.0 \%)$.

Esses estudantes estão concentrados na faixa etária de 20 a 24 anos $(n=341 ; 60,1 \%)$, predominantemente solteiros ( $n=549 ; 96,8 \%)$, sem filhos ( $n=537 ; 94,5 \%)$, em sua maioria do gênero feminino $(n=444 ; 78.3 \%)$. Desses estudantes, 305 (53,7\%) moram com colegas/ amigos em repúblicas ou pensionatos.

Tabela 1- Caracterização acadêmica e sociodemográfica dos universitários entrevistados. Diamantina, MG, Brasil, 2014

\begin{tabular}{|c|c|c|}
\hline Variáveis & $\mathbf{N}$ & Frequência (\%) \\
\hline \multicolumn{3}{|l|}{ Cursos } \\
\hline Ciências Biológicas & 76 & $13,4 \%$ \\
\hline Educação Física & 73 & $12,9 \%$ \\
\hline Enfermagem & 49 & $8,6 \%$ \\
\hline Farmácia & 102 & $18,0 \%$ \\
\hline Fisioterapia & 98 & $17,3 \%$ \\
\hline Nutrição & 67 & $11,8 \%$ \\
\hline Odontologia & 102 & $18,0 \%$ \\
\hline Total & 567 & $100 \%$ \\
\hline \multicolumn{3}{|l|}{ Gênero } \\
\hline Feminino & 444 & $78,3 \%$ \\
\hline Masculino & 123 & $21,7 \%$ \\
\hline Total & 567 & $100 \%$ \\
\hline \multicolumn{3}{|l|}{ Faixa etária } \\
\hline 15 a 19 & 127 & $22,4 \%$ \\
\hline 20 a 24 & 341 & $60,1 \%$ \\
\hline 25 a 29 & 85 & $15,0 \%$ \\
\hline 30 ou mais & 14 & $2,5 \%$ \\
\hline Total & 567 & $100 \%$ \\
\hline \multicolumn{3}{|l|}{ Estado civil } \\
\hline Solteiro & 549 & $96,8 \%$ \\
\hline Casado/ "Vive junto" & 15 & 2,7 \\
\hline Separado & 3 & 0,5 \\
\hline Viúvo & 0 & $0,0 \%$ \\
\hline Total & 567 & $100 \%$ \\
\hline \multicolumn{3}{|l|}{ Mora com } \\
\hline Pais/familiares & 205 & $36,2 \%$ \\
\hline Cônjuge/Companheiro & 22 & $3,9 \%$ \\
\hline Amigos/República/Pensão & 305 & $53,7 \%$ \\
\hline Sozinho & 29 & $5,1 \%$ \\
\hline Outros & 6 & $1,1 \%$ \\
\hline Total & 567 & $100 \%$ \\
\hline \multicolumn{3}{|l|}{ Possuem filhos } \\
\hline Sim & 30 & $4,5 \%$ \\
\hline Não & 537 & $94,5 \%$ \\
\hline Total & 567 & $100 \%$ \\
\hline
\end{tabular}

Um somatório de 11,6\% $(n=66)$ dos estudantes declarou nunca ter experimentado substâncias psicoativas na vida. Dos 567 estudantes, 168 (29,62\%) experimentaram drogas ilícitas antes de entrar na universidade (Tabela 2).

Comparando o consumo das substâncias por cursos, a maior taxa de universitários que nunca experimentaram as substâncias encontra-se nos cursos de Farmácia $(n=13)$ e Odontologia $(n=13)$ (Tabela 2).

Já como acadêmicos, 501 entrevistados declararam ter usado álcool e 161 usaram tabaco, dentre as drogas lícitas. Em relação às ilícitas, a mais frequente foi a maconha, usada por 115 estudantes $(20,3)$, sendo a maioria do curso de Odontologia $(n=30)$, que também mostrou a maior parte dos usuários de tabaco $(40,2 \%)$, maconha $(29,4 \%)$, cocaína $(5,9 \%)$, crack $(1,9 \%)$, inalantes $(26,5 \%)$, alucinógenos $(18,6 \%)$ e ecstasy $(14,7 \%)$ (Tabela 3$)$. No curso de Fisioterapia, encontrou-se a maior proporção dos que já experimentaram álcool $(92,9 \%)$ e tranquilizantes $(20,4 \%)$ (Tabela 3).

Os percentuais por curso referem-se ao " $n$ " de cada curso em separado.

Quanto à idade de início de uso de substâncias psicoativas, predominou a faixa etária de 15 a 19 anos, exceto a cocaína, que predomina a faixa etária de 20 a 24 anos. Mais detalhes quanto ao uso de substâncias psicoativas lícitas (álcool e tabaco) se encontram na Figura 1 que distingue, por curso, as frequências de uso na vida, nos últimos 12 meses e nos últimos 30 dias da entrevista (Figura 1).

Como pode ser visto na Figura 2, encontrou-se associação de bebidas alcoólicas com outras substâncias, pelo menos uma vez na vida, no último ano e no último mês. As maiores frequências foram para universitários do curso de Odontologia, do gênero feminino (69,6\%, $70,2 \%, 63,2 \%)$, com idade entre 20 e 24 anos $(65,4 \%$, $68,2 \%, 70,8 \%)$.

Com relação aos principais motivos que levaram os entrevistados a experimentar as substâncias pela primeira vez, destaca-se a curiosidade como principal fator, tanto para o sexo masculino $(40,4 \%)$ quanto para o feminino $(46,6 \%)$, seguida de diversão e prazer (Homens: 35,1\%; mulheres: 25,0\%) e alívio da tensão psicológica (Homens: 1,8\%; Mulheres: $8,7 \%$ ).

Referente às principais influências que levaram ao consumo de drogas pela primeira vez, os entrevistados de todos os cursos indicaram os amigos, com as seguintes porcentagens: 90,9\% (Nutrição), 83,8 \% (Odontologia), $80,0 \%$ (Fisioterapia), 75,0\% (enfermagem), 67,6\% (Farmácia), 61,1\% (Ciências Biológicas) e 57,9\% (Educação Física). 
Tabela 2 - Frequência de uso das substâncias psicoativas pelos estudantes antes de entrar na universidade por gênero e curso. Diamantina, MG, Brasil, 2014

\begin{tabular}{|c|c|c|c|c|c|c|c|c|c|c|c|}
\hline & \multirow{2}{*}{ Substâncias } & \multicolumn{2}{|c|}{ Gênero } & \multicolumn{7}{|c|}{ Curso } & \multirow{2}{*}{ Total } \\
\hline & & $\mathrm{F}^{*}$ & $\mathbf{M}^{\dagger}$ & $\mathrm{CB}^{\ddagger}$ & $\mathrm{EF}^{\S}$ & EN" & $\mathrm{FA}^{\pi}$ & $\mathrm{Fl}^{*+}$ & $\mathrm{NU}^{+t}$ & $O D^{\ddagger \ddagger}$ & \\
\hline & $N S^{\S \S}$ & 51 & 15 & 11 & 11 & 04 & 13 & 07 & 07 & 13 & 66 \\
\hline \multirow{2}{*}{ Lícitas } & Tabaco & -1111 & -ll1! & 63 & 67 & 28 & 91 & 82 & 46 & 124 & 501 \\
\hline & Álcool & -1111 & $-^{1111}$ & 64 & 62 & 45 & 89 & 91 & 60 & 90 & 501 \\
\hline \multirow{13}{*}{ Ilícitas } & Maconha & 27 & 28 & 6 & 11 & 2 & 9 & 8 & 4 & 15 & 55 \\
\hline & Alucinógenos & 4 & 8 & 0 & 3 & 1 & 1 & 1 & 0 & 6 & 12 \\
\hline & Cocaína & 4 & 9 & 1 & 2 & 0 & 2 & 2 & 2 & 4 & 13 \\
\hline & Crack & 0 & 1 & 0 & 0 & 0 & 0 & 0 & 0 & 1 & 1 \\
\hline & Anfetamínicos & 4 & 2 & 1 & 0 & 1 & 3 & 1 & 0 & 0 & 6 \\
\hline & Anticolinérgicos & 2 & 3 & 0 & 2 & 0 & 1 & 1 & 0 & 1 & 5 \\
\hline & Inalantes & 14 & 17 & 0 & 5 & 4 & 7 & 4 & 0 & 11 & 31 \\
\hline & Tranquilizantes & 9 & 6 & 2 & 0 & 3 & 3 & 2 & 1 & 4 & 15 \\
\hline & Opiáceos & 2 & 1 & 1 & 0 & 1 & 0 & 1 & 0 & 0 & 3 \\
\hline & Barbitúricos & 1 & 1 & 0 & 0 & 0 & 0 & 1 & 0 & 1 & 2 \\
\hline & Sintéticas & 0 & 1 & 0 & 1 & 0 & 0 & 0 & 0 & 0 & 1 \\
\hline & Ecstasy & 7 & 8 & 0 & 4 & 1 & 2 & 2 & 2 & 4 & 15 \\
\hline & Outras & 3 & 6 & 0 & 1 & 1 & 3 & 1 & 1 & 2 & 9 \\
\hline \multicolumn{2}{|c|}{ Total para drogas ilícitas } & 77 & 91 & 11 & 29 & 14 & 31 & 24 & 10 & 49 & 168 \\
\hline
\end{tabular}

*feminino; ${ }^{\dagger}$ masculino; ${ }^{\ddagger} \mathrm{CB}=$ Ciências Biológicas; ${ }^{\mathrm{S}} \mathrm{EF}=$ Educação Física; "EN = Enfermagem; ${ }^{\mathrm{F} A}=$ Farmácia; ${ }^{* *} \mathrm{FI}=$ Fisioterapia; ${ }^{+} \mathrm{NU}=\mathrm{Nutrição;}$ ${ }^{\ddagger} \mathrm{OD}=$ Odontologia; ${ }^{\S \S}$ Nunca usaram nenhuma substância; "'IO questionário não abordou tais dados

Tabela 3 - Frequência do uso das substâncias psicoativas depois de entrar na universidade segundo o curso. Diamantina, MG, Brasil, 2014

\begin{tabular}{|c|c|c|c|c|c|c|c|c|}
\hline Substância & $\begin{array}{l}C^{*} \\
n(\%)\end{array}$ & $\begin{array}{c}E F^{\dagger} \\
n(\%)\end{array}$ & $\begin{array}{c}\mathrm{EN}^{\ddagger} \\
\mathrm{n}(\%)\end{array}$ & $\begin{array}{c}\mathrm{FA}^{\S} \\
\mathrm{n}(\%)\end{array}$ & $\begin{array}{c}F^{\prime \prime} \\
n(\%)\end{array}$ & $\begin{array}{l}N U^{* *} \\
n(\%)\end{array}$ & $\begin{array}{l}O D^{t t} \\
n(\%)\end{array}$ & $\begin{array}{c}\text { Total } \\
\mathbf{n}\end{array}$ \\
\hline Álcool & $64(84,2)$ & $45(91,8)$ & $62(84,9)$ & $89(87,3)$ & $91(92,9)$ & $60(89,6)$ & $89(87,4)$ & 501 \\
\hline Tabaco & $21(27,6)$ & $5(10,2)$ & $22(30,1)$ & $30(29,4)$ & $27(27,5)$ & $15(22,4)$ & $41(40,2)$ & 161 \\
\hline Maconha & $17(22,3)$ & $7(14,2)$ & $16(21,9)$ & $21(20,6)$ & $16(16,3)$ & $8(12,0)$ & $30(29,4)$ & 115 \\
\hline Cocaína & $2(2,6)$ & - & $8(10,9)$ & $5(4,9)$ & $2(2,04)$ & $3(4,5)$ & $6(5,9)$ & 26 \\
\hline Crack & $1(1,3)$ & - & - & - & - & - & $2(1,9)$ & 01 \\
\hline Tranquilizantes & $9(11,7)$ & $10(20,4)$ & $5(6,8)$ & $16(15,6)$ & $20(20,4)$ & $6(9,0)$ & $20(19,6)$ & 86 \\
\hline Inalantes & $4(5,2)$ & $9(18,3)$ & $9(12,3)$ & $18(17,6)$ & $10(10,2)$ & $8(12,0)$ & $27(26,5)$ & 85 \\
\hline Alucinógenos & - & $2(4,0)$ & $6(5,6)$ & $7(6,8)$ & $3(3,06)$ & $2(2,9)$ & $19(18,6)$ & 39 \\
\hline Ecstasy & $1(1,3)$ & $2(4,0)$ & $7(9,5)$ & $4(3,9)$ & $4(4,08)$ & $3(4,5)$ & $15(14,7)$ & 36 \\
\hline
\end{tabular}

${ }^{*} \mathrm{CB}=$ Ciências Biológicas; ${ }^{\dagger} \mathrm{EF}=$ Educação Física; ${ }^{\circledR} \mathrm{EN}=$ Enfermagem; ${ }^{\circledR} \mathrm{FA}=$ Farmácia; ${ }^{\mathrm{F} I}=$ Fisioterapia; $* * \mathrm{NU}=\mathrm{Nutrição} ;{ }^{+\dagger} \mathrm{OD}=\mathrm{Odontologia}$

Quando questionados a respeito dos conhecimentos sobre drogas, a maioria dos estudantes $(47,4 \%$; $\mathrm{n}=269)$ se considera muito bem informada. Entretanto, $17,1 \%(n=97)$ dos estudantes gostariam de obter mais informações sobre as substâncias psicoativas e $25,7 \%$ $(n=146)$ apenas gostariam de ajudar pessoas com problemas relacionadas às drogas, já 40,6\% $(n=230)$ referiram as duas opções anteriores. Em relação aos meios de se obter informações sobre álcool e outras drogas, a Internet $(54,9 \% ; n=311)$ foi a opção mais escolhida, seguida dos pais $(13,8 \% ; n=78)$ e amigos $(13,4 \% ; n=76)$.

Ao realizar o cruzamento dos dados, verificou-se que houve uma associação significativa entre o uso de substâncias psicoativas em relação ao gênero após o ingresso na universidade $(p=0,014$, Teste qui-quadrado de Pearson), sendo mais prevalente no gênero feminino, que de um total de $425,80,7 \%$ fizeram uso de SPA $(n=344)$, ao passo que dentre os homens $(n=116)$, apenas $69,8 \%$ deles usaram SPA $(n=81)$. 

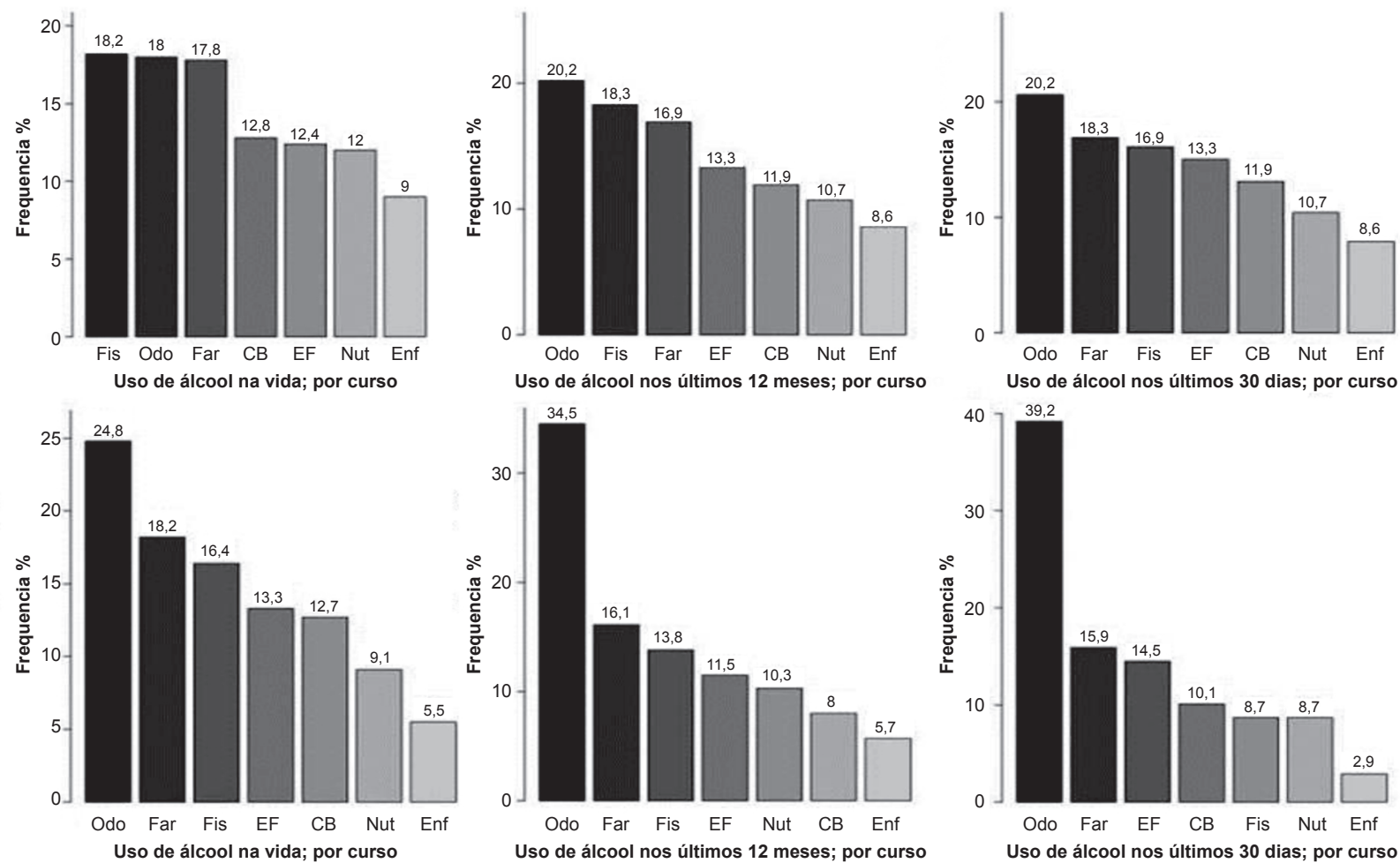

Uso de álcool nos últimos 12 meses; por curso

Uso de álcool nos últimos 30 dias; por curso

Figura 1 - Uso do álcool e tabaco na vida, nos últimos 12 meses e nos últimos 30 dias, por curso

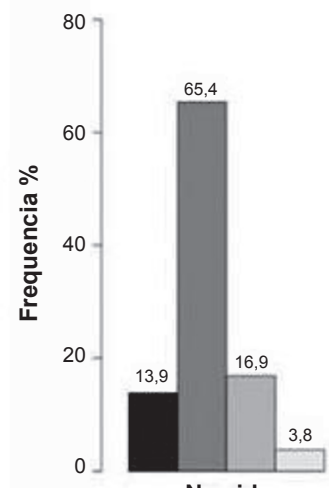

Na vida

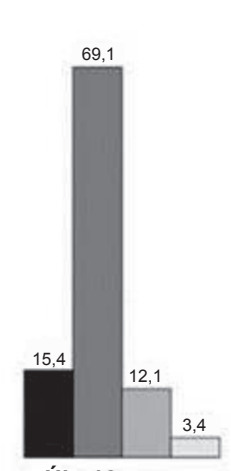

Últ. 12 meses

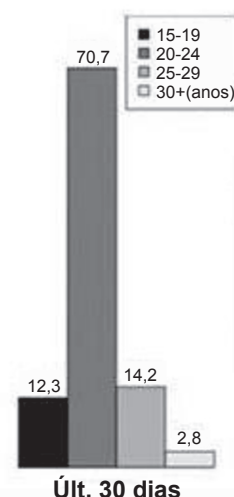

Últ. 30 dias
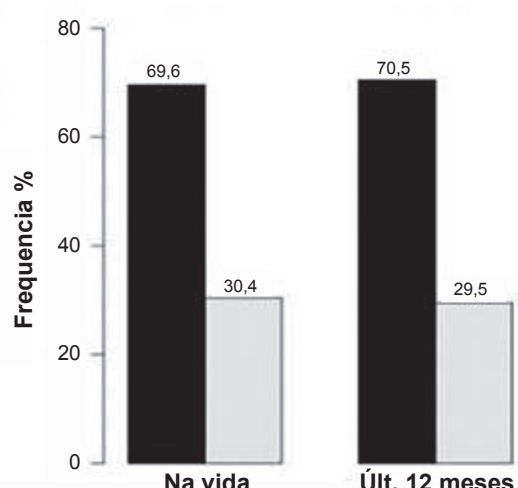

Últ. 12 meses

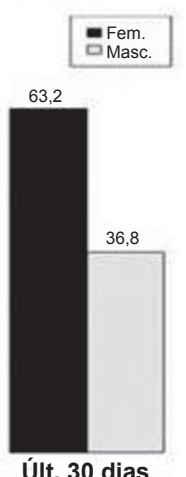

Últ. 30 dias

Figura 2 - Associação do álcool com outras substâncias na vida, no ano e no mês, por gênero e faixa etária

\section{Discussão}

Ao realizar a comparação entre os gêneros, encontrou-se prevalência significativamente maior no uso de substâncias psicoativas entre as mulheres após a entrada na universidade ( $p=0,014$, Teste Qui-quadrado de Pearson), o que corrobora os achados de outros pesquisadores ${ }^{(1,8)}$. No levantamento realizado entre os universitários das 27 capitais brasileiras, o uso múltiplo e simultâneo de álcool a outras substâncias foi relatado, com maior prevalência, entre os homens e entre os jovens de 25 a 34 anos de idade, resultados contrários foram observados no nosso trabalho, no qual prevaleceu o sexo feminino com idade ente 20 e $24 \operatorname{anos}^{(8)}$.

A associação significativa do consumo de SPA no gênero feminino $(p=0,014)$ difere dos resultados relatados por autores que evidenciaram consumo maior no gênero masculino em estudo realizado com estudantes da Universidade de Guayaquil(11); também em uma revisão de literatura sobre o abuso de álcool e drogas como indicadores de comportamentos violentos, que além de encontrar maior prevalência entre os homens, encontrou uma associação entre o uso abusivo e a violência(12). Além disso, as justificativas para uso de SPA também diferem, sendo que as mulheres as usam como forma de ajudar a enfrentar problemas ${ }^{(13)}$, enquanto que os homens as utilizam para ficar "altos" e mudar seu comportamento, ficando levemente embriagados ${ }^{(11,14)}$. Em relação à idade na qual se iniciou o uso de SPA, houve o predomínio da faixa etária de 20 a 24 anos; em outras pesquisas, predomina a faixa etária de 18 a $24 \operatorname{anos}^{(8)}$. 
Observou-se neste estudo que o consumo de drogas lícitas foi o mais prevalente, como o álcool $(88,4 \%)$, o que foi semelhante ao encontrado no I Levantamento Nacional sobre o Uso de Álcool, Tabaco e outras drogas entre universitários das 27 capitais brasileiras $(86,7 \%)$ (9), bem como os índices relatados em trabalhos semelhantes ${ }^{(5,9)}$.

Esse manuscrito aponta o consumo da maconha antes do ingresso na universidade, como observado por outros pesquisadores ${ }^{(8)}$, que também verificaram o mesmo padrão de uso. Em contrapartida, um trabalho realizado em Feira de Santana com indivíduos de 12 a 24 anos evidencia o início do consumo de substâncias psicoativas até 14 anos de idade, predominando o consumo de tabaco, maconha, cocaína e crack. Naqueles que iniciaram o consumo após os 14 anos, constatou-se o uso precoce de crack entre adolescentes na faixa dos 15 a 19 anos $^{(15)}$.

Dentre os motivos para a experimentação de drogas pela primeira vez, a curiosidade foi o mais prevalente, seguido pela diversão e prazer, concordando em parte com o estudo realizado em uma universidade particular de Curitiba, na qual o principal motivo foi a busca de diversão ou prazer(3), e também com outro realizado na Pontifícia Universidade de São Paulo, em que encontraram o uso recreacional como o principal motivo para o consumo de drogas nos $\operatorname{cursos}^{(16)}$. Os amigos ou conhecidos são apontados como os maiores incentivadores para introdução ao uso e como companhia frequente para o consumo de substâncias psicoativas $^{(1,17)}$, resultados que corroboram os nossos achados.

O ingresso na universidade traz uma série de mudanças de caráter pessoal aos estudantes, em especial para os que não residem na mesma cidade em que a universidade se localiza. A saída da casa dos pais traz novas responsabilidades ligadas a tarefas cotidianas, impulsionando o desenvolvimento da autonomia, exigindo o desenvolvimento de respostas adaptativas perante um conjunto de situações desafiadoras relacionadas ao gerenciamento da própria vida(18), o que incontestavelmente gera estresse e ansiedade, e esse fato encontra suporte em nosso estudo, no qual os entrevistados admitiram que o alívio da tensão psicológica foi um dos motivos para terem experimentado SPA. Uma pesquisa realizada com acadêmicos de uma universidade da região norte do Rio Grande do Sul também destaca que o maior desafio dos estudantes é absorver novas responsabilidades(19). Em meio a tantas mudanças e situações estressantes, é importante destacar o papel da família na prevenção ao uso de drogas por jovens, por meio da adequada transmissão de valores, monitoramento e imposição de limites. Nesse ponto, a universidade teria uma responsabilidade a cumprir, visto que para frequentá-la muitos jovens saem do seio de suas famílias(20), dado corroborado em nosso estudo, em que a maioria dos entrevistados $(53,7 \%)$ mora em repúblicas, em pensões ou com amigos; e a influência dos mesmos é perceptível, como descrita em investigações recentes, fundamentada na premissa de que jovens procuram seguir o comportamento do grupo no qual estão inseridos, buscando, dessa forma, status e a sensação de pertencimento, motivados pela busca da diversão, prazer, quebra de rotina e redução da ansiedade e estresse ${ }^{(21)}$.

Os universitários pesquisados indicaram, em sua maioria, estarem bem $(47,4 \%)$ ou muito bem informados $(46,6 \%)$ a respeito das substâncias psicoativas, o que talvez pudesse ter contribuído para uma prevalência menor no uso das SPA, visto que a informação é tida como um dos principais fatores protetores ao uso de drogas ${ }^{(22)}$ e também é importante elemento na elaboração de programas de prevenção(23); em contrapartida, contrariando esses conceitos, em uma pesquisa realizada com estudantes do ensino médio no México, encontrou-se que a probabilidade de usar drogas é até três vezes maior com o aumento da escolaridade, pois, se por um lado há a formação educativa, por outro podem estar se desvinculando dos familiares, associando-se a grupos de amigos, condições favoráveis para o envolvimento com fatores de risco como experimentar drogas ${ }^{(24)}$, fatores que talvez possam explicar o alto índice encontrado em nesta pesquisa, visto que a maioria dos entrevistados $(53,7 \%)$ mora fora de casa, com amigos, em repúblicas ou pensionatos.

Os participantes do estudo, quando questionados sobre o primeiro recurso que procurariam em caso de dúvidas sobre drogas, em sua maioria, citaram a internet $(54,9 \%)$ como o meio de obter informações acerca do assunto e isso pode ter contribuído para a alta prevalência no uso de SPA, já que um trabalho realizado com estudantes do curso de Educação Física em uma universidade pública do Maranhão afirma que o consumo de substâncias psicoativas é frequentemente estimulado pela mídia, como em anúncios comerciais, filmes, letras de músicas e outros meios de comunicação de massa. A exposição contínua a essas substâncias associadas a fatores desejáveis, como prazer, beleza, sucesso financeiro, sexual e outros, de forma explícita ou implícita, configura-se em um importante fator de risco para o consumo vicioso(25).

As limitações desta pesquisa estão relacionadas com a amostra que não foi representativa, por se tratar de uma amostra de conveniência e não haver pareamento entre os gêneros, nem proporcionalidade entre os cursos de acordo com o número de estudantes.

Este estudo pretende contribuir no sentido de alertar a população universitária com relação aos 
prejuízos inerentes ao consumo alcoólico exacerbado e destacar a necessidade de se trabalhar o tema com vistas a prevenir os problemas ora mencionados.

\section{Conclusão}

Os resultados do presente estudo revelam que o uso de substâncias psicoativas entre os estudantes dos cursos de Ciências Biológicas e da saúde na Universidade Federal dos Vales do Jequitinhonha e Mucuri é expressivo, tornando-se fator preocupante no que diz respeito à saúde dessa população.

Esses resultados apontam a necessidade de intervenção, principalmente entre os estudantes do curso de Odontologia, servindo como ponto de partida para desenvolver projetos na área da prevenção ao uso abusivo de substâncias psicoativas pela abordagem dessa temática no currículo acadêmico dos estudantes da UFVJM e a criação de futuros programas específicos de prevenção para os universitários.

\section{Referências}

1 Chiapetti N, Serbena CA. Alcohol, Tobacco and other Drugs used by Students of Health Services from a University in Curitiba. Psicol Reflex Crit. 2007;20(2):303-13. doi: 10.1590/ S0102-79722007000200017

2. Garcia VM, Costa ML Jr. Illegal drug consumption and the relation with the environment. SMAD, Rev Eletrônica Saúde Mental Álcool Drog. [Internet]. 2016;12(1):3-11. [cited Sep 6 2017]. Available from: <http://pepsic. bvsalud.org/pdf/smad/v12n1/02.pdf >.

3. Peuker AC, Fogaça J, Bizarro L. Expectations and Problematic Drinking among College Students. Psicol Teoria Pesq. 2006 Mai/Ago;22(2):193-200. doi: 10.1590/S0102-37722006000200009

4. Carvalho AMP, Cunningham J, Strike C, Brands B, Wright MGM. Perceived norms among university students of three health courses for drug use among peers. Rev. Latino-Am. Enfermagem. 2009 Nov/Dez;17(Esp):900-906. doi: 10.1590/ S0104-11692009000700022

5. Lemos KM, Neves NMBC, Kuwano AY, Tedesqui G, Bitencourt AGV, Neves FBCS, et al. Psychoactive substance use by medical students from Salvador (BA). Rev Psiquiatr Clín. 2007;34(3):118-24. doi: 10.1590/ S0101-60832007000300003

6. Wagner GA, Andrade AG. The use of alcohol, tobacco and other drugs among Brazilian college students. Rev Psiquiat Clín. 2008;35(Suppl1):48-54. doi: 10.1590/ S0101-60832008000700011

7.Pereira DS, Souza RS, Buaiz V, Siqueira MM. Psychoactive substance use among Medicine students from Espirito Santo Federal University.
J Bras Psiquiatr. 2008;57(3):188-95. doi: 10.1590/ S0047-20852008000300006

8. Mardegan PS, Souza RS, Buaiz V, Siqueira MM. Psychoactive substance use between students of the nursing. J Bras Psiquiatr. 2007;56(4):260-6. doi: 10.1590/S0047-20852007000400004

9. Portugal FB, Souza RS, Buaiz V, Siqueira MM. Use of drugs by Pharmacy students at the Federal University in Espírito Santo. J Bras Psiquiatr. [Internet]. 2008;57(2):12732. [cited August 29 2017]. Available from: <http://www. scielo.br/pdf/jbpsiq/v57n2/a08v57n2.pdf>.

10. Andrade AG, Duarte PCAV, Oliveira LG. I levantamento nacional sobre o uso de álcool, tabaco e outras drogas entre universitários das 27 capitais brasileiras. Brasília: Secretaria Nacional de Políticas sobre Drogas; 2010. v.1, 284 p.

11. Chavez KAP, O'Brien B, Pillon SC. Drugs use and risk behavior in a university community. Rev. Latino-Am. Enfermagem. 2005 Nov/Dec;13(Esp):1194-200. doi: 10.1590/S0104-11692005000800014

12. Friedman AS. Substance use/abuse as a predictor to illegal and violent behavior: A review of the relevant literature. Aggress Violent Behav. 1998;3(4):339-55. doi: 10.1016/S1359-1789(97)00012-8

13. Kauffman SE, Silver P, Poulin J. Gender differences in attitudes toward alcohol, tobacco, and other drugs. Soc Work. 1997;42(3):231-41. doi: 10.1093/sw/42.3.231

14. Çırakoğlu OC, Işın G. Perception of drug addiction among Turkish university students: Causes, cures, and attitudes. Addict Behav. 2005;30(1):1-8. doi: 10.1016/j. addbeh.2004.04.003

15. Silva CC, Costa MCO, Carvalho RC, Amaral MTR, Cruz NLA, Silva MR. Initiation and consumption of psychoactive substances among adolescents and young adults in an Anti-Drug Psychosocial Care Center. Cienc Saúde Coletiva. 2014;19(3):737-45. doi: 10.1590/1413-81232014193.15922013

16. Martinho AF, Tonin CL, Nunes LM, Novo NF, Hübner CVK. Alcohol and drug use among nursing, biology and medical students from Pontifícia Universidade Católica de São Paulo. Rev Fac Ciênc Méd Sorocaba. [Internet]. 2009;11(1):11-5. [cited Sep 5 2017]. Available from: https://revistas.pucsp.br/index.php/RFCMS/article/ view/1814/1142.

17. Rocha PR, David HMSL. Patterns of alcohol and drug consumption in health care professionals: a portrait of students of lato sensu courses in a public institution. SMAD, Rev Eletrônica Saúde Mental Álcool Drog. [Internet]. 2015;11(1):42-8. [cited Sep 5 2017]. Available from: <http://pepsic. bvsalud.org/scielo.php?script =sci_abstract\&pi $\mathrm{d}=$ S1806-69762015000100007>.

18. Teixeira MAP, Dias ACG, Wottrich SH, Oliveira AM. Adaptation to university among young freshmen 
students. Psicol Esc Educ. 2008;12(1):185-202. doi: 10.1590/S1413-85572008000100013

19. Cervinski L, Enricone J. Perception of college freshmen on the process of adaptation after leaving parental house. Perspectiva. [Internet]. 2012;36(136):101-10. [cited Sep 5 2017]. Available from: < http://www.uricer. edu.br/site/pdfs/perspectiva/136_311.pdf>.

20. Oliveira EB, Bittencourt LP, Carmo AC. The importande of family in drug use prevention among children and adolescents: mothering role. SMAD, Rev Eletrônica Saúde Mental Álcool Drog. [Internet]. 2008;4(2):1-16. [cited Sep 5 2017]. Available from: <http://pepsic.bvsalud.org/scielo.php?script=sci_artte xt\&pid=S1806-69762008000200003>.

21. Garcia JJ, Pillon SC, Santos MA. Relations between family context and substance abuse in high school adolescents. Rev. Latino-Am. Enfermagem. 2011;19(Esp):753-761. doi: 10.1590/ S0104-11692011000700013

22. Sanchez ZVDM, Oliveira LG, Nappo SA. Protective factors from adolescents against drug use emphasizing the role of religiosity. Ciênc Saúde Coletiva. 2004;9(1):43-55. doi: 10.1590/ S1413-81232004000100005

23. Robertson EB, David SL, Rao SA. Preventing drug use among children and adolescents: A research-based guide for parents, educators, and community leaders. 2nd ed. Maryland: National Institute on Drug Abuse; 2003. $41 \mathrm{p}$.

24. Jinez MLJ, Souza JRM, Pillon SC. Drug use and risk factors among secondary students. Rev. Latino-Am. Enfermagem. 2009 Mar/Abr;17(2):246-52. doi: 10.1590/S0104-11692009000200017

25. Costa JJ Júnior, Gomes RIB, Sousa VEC, Sardinha AHL, Viveiros MTM. Alcohol consuption among academics of Physical Education. Rev RENE. 2012;13(2):3861-95. doi: 10.15253/rev\%20rene. v13i2.3933 